\title{
Experimental Strategies for the Identification of DNA-Binding Proteins
}

\author{
Michael F. Carey, Craig L. Peterson, and Stephen T. Smale
}

\begin{abstract}
This article describes approaches for identifying proteins that bind conserved or functional DNA motifs. It discusses the use of consensus sequence databases to identify candidate proteins capable of binding a DNA motif of interest and then explains the potential uses of sophisticated mass spectrometry technology. DNA-binding proteins are most commonly identified by electrophoretic mobility-shift assay (EMSA) or DNase I footprinting. Each of these methods is described, and their advantages and limitations are outlined. It is important to stress that each of the strategies discussed in this article may identify one or more proteins that bind a DNA element of interest. However, none of the strategies will necessarily lead to the protein that is the functionally relevant regulator of the control element in the context of the endogenous locus. Regardless of how a DNA-binding protein is isolated and identified, it merely becomes a candidate regulator of the gene of interest.
\end{abstract}

Database methods can be used as a means of identifying transcription factors that could interact with a DNA motif or control region of interest. The two most commonly used databases as of this writing, TRANSFAC (http://www.gene-regulation.com/pub/databases.html) and JASPAR (http:// jaspar.genereg.net/), are designed to allow a researcher to determine whether a DNA sequence motif corresponds to a potential recognition site for a previously characterized protein. Unfortunately, these databases contain high-quality information only for those proteins whose binding sites have been defined in rigorous experimental studies. Furthermore, information about the range of DNA sequences that can support functional interactions with a DNA-binding protein is incomplete, even for the best-characterized DNA-binding proteins. Therefore, there remains a reasonably high probability that the relevant protein, and all other members of the same protein family, will be missed in a database search.

The parameters used for binding-site database searches can and should be varied. The searches can be performed using high-stringency parameters, in which only perfect matches to reported consensus sequences will be revealed. Search parameters can then be adjusted to allow mismatches to the consensus at one or more random or specific positions. It is strongly recommended that the analysis be performed with a variety of parameters to identify as many candidate proteins as possible.

If the DNA motif of interest contains a perfect match to a consensus sequence, the predicted binding protein may be responsible for the function of the motif, but not necessarily. One possibility is that multiple proteins are capable of binding the isolated site in vitro, but only one of these proteins, perhaps one not identified in the database analysis, is responsible for the element's function in vivo. A second possibility is that the consensus sequence is not entirely accurate: The consensus sequences

Adapted from Transcriptional Regulation in Eukaryotes: Concepts, Strategies, and Techniques, 2nd edition, by Michael F. Carey, Craig L. Peterson, and Stephen T. Smale. CSHL Press, Cold Spring Harbor, NY, USA, 2009.

(C) 2012 Cold Spring Harbor Laboratory Press

Cite this article as Cold Spring Harbor Protoc; 2012; doi:10.1101/pdb.top067470 
used to create databases such as TRANSFAC and JASPAR generally have not been verified by multiple investigators and often are derived from data reported in a single study. Third, the consensus sequence included in the database may be incomplete. For example, consensus sequences of only 4 or 5 bp are represented in the databases for some factors, even though high-affinity binding by these factors relies on a longer sequence. Consensus sequences are sometimes incomplete because, for some factors, it is very difficult to determine which combinations of base pairs surrounding a 4-bp core sequence, for example, are necessary for high-affinity binding. Ultimately, the quality of databases such as TRANSFAC and JASPAR is entirely dependent on the quality of the experimental data used to assemble them.

Database searches performed using a variety of parameters will allow a researcher to identify a collection of DNA-binding proteins that may recognize the sequence of interest. At this point, the researcher should carefully consult the primary literature to evaluate the quality of the studies used to define the consensus sequence for each candidate protein and the degree to which the consensus sequence may be complete or incomplete. Binding-site selection studies used to define consensus recognition sequences are often biased toward the highest-affinity binding sites and can miss DNA motifs that interact with the protein with only moderately reduced affinity, despite the fact that these loweraffinity interactions could be of equal functional significance. In fact, functional recognition sequences for some proteins frequently interact with low affinity, because of the need for cooperative binding with another protein bound to a nearby site.

This review of the literature should not be restricted to studies that attempted to define consensus recognition sequences. It should also include a careful evaluation of structural studies of the proteinDNA interaction. The structure of the protein-DNA complex determined by X-ray crystallography or NMR (nuclear magnetic resonance) might reveal critical contacts between particular residues of the protein's DNA-binding domain and specific bases of the recognition sequence. This information can help determine the validity of the experimentally defined consensus sequence and the importance of specific base pairs within the consensus.

After a collection of proteins is identified that may interact with the DNA motif, a number of other considerations can help lead to the most attractive candidates. One criterion is the precise DNA sequence found to be conserved through evolution or the precise sequence required for functional activity of the control region in transfection experiments. If the nucleotides found to be important for the protein-DNA interaction correspond closely to those that are conserved through evolution or that are required for functional activity, confidence that the candidate protein is relevant for the function of the element increases. Conversely, if the key base pairs required for protein binding are not conserved through evolution or do not coincide with functionally important base pairs, the researcher could expect that the candidate protein might not be functionally relevant.

Another important criterion is whether the candidate protein is expressed in appropriate cell types, at appropriate stages of development, and/or in response to appropriate stimuli. If the protein is not expressed in cells that express the target gene, the information may nevertheless be valuable, as another member of the same protein family that recognizes similar DNA sequences may instead be responsible. Complete genome sequences for a variety of organisms, as well as the ease of obtaining expression data, can be extremely valuable in this regard. If a database analysis predicts that a particular protein interacts with a DNA motif, it is now quite easy to identify all members of the family with homologous DNA-binding domains that might interact with similar sequences. Furthermore, microarray experiments can readily be performed to identify the family members expressed in the cells of interest. Although knowledge that multiple members of a family recognize similar sequences can be of considerable value, this fact can make it difficult to determine precisely which family member or members are responsible for the element's function.

Finally, in addition to evaluating (1) the literature, (2) the base pairs conserved through evolution, (3) the base pairs required for the element's function, and (4) the expression data, EMSA and chromatin immunoprecipitation (ChIP) experiments can be performed to determine whether the predicted protein is indeed capable of binding the DNA motif in vitro and associating with the DNA in the vicinity of the motif in vivo (see below). 
M.F. Carey et al.

\section{MudPIT ANALYSIS OF DNA-BINDING PROTEINS}

If it becomes appropriate to search for DNA-binding proteins capable of recognizing a motif of interest beyond those predicted to interact in a database search, most of the conventional strategies described later in this article have been successful in numerous studies. However, newer strategies that rely on sophisticated mass spectrometry technologies are beginning to emerge as viable alternatives that may ultimately replace the conventional strategies. For example, it may be possible to use relatively simple pulldown experiments in conjunction with multidimensional protein identification technology (MudPIT) analysis (Washburn et al. 2001; Fournier et al. 2007) to identify rapidly a collection of proteins capable of binding a sequence motif in a cell extract.

Historically, it has been difficult to isolate eukaryotic proteins capable of binding DNA motifs of interest and to clone the genes encoding those proteins. The most common strategy has been to purify proteins by sequence-specific DNA affinity chromatography. Before attempting purification, it generally is important to develop a protein-DNA interaction assay to detect the protein. For affinity chromatography, DNA fragments or oligonucleotides containing the DNA motif of interest are often ligated into tandem arrays and linked covalently to a resin. Nuclear extracts or fractions from prior conventional chromatography columns are then applied to the affinity column, followed by washing and elution. Originally, Edman degradation was used to obtain a partial amino acid sequence of the pure protein observed by sodium dodecyl sulfate polyacrylamide gel electrophoresis (SDSPAGE), leading to cDNA cloning by library screening using a degenerate DNA probe. Several years ago, mass spectrometry largely replaced Edman degradation for identifying purified DNA-binding proteins.

In most instances, this conventional protein purification strategy has proved to be extremely challenging. Typical transcription factors are moderately abundant (in the range of 250,000 copies per cell), but they often are extracted inefficiently into nuclear extracts. It is therefore often difficult to purify sufficient quantities of protein for detection on a stained SDS-PAGE gel. In addition, cells contain abundant DNA- and RNA-binding proteins that bind DNA affinity columns readily but with limited specificity. These nonspecific nucleic acid-binding proteins make it difficult to identify transcription factors that bind to the column in a sequence-specific manner. To help overcome this difficulty, researchers generally add nonspecific competitor DNA to the extract before loading the column. Conventional column chromatography steps before DNA affinity chromatography can also help separate the protein of interest from nonspecific binding proteins. Including substantial amounts of competitor DNA reduces yield during the affinity chromatography step because much of the desired protein binds to the competitor rather than the affinity column. The reduced yield can be overcome by increasing the amount of starting material. Because of these challenges (and others described below), purifying DNA-binding proteins can require one or more years of effort, as well as considerable protein purification expertise.

Importantly, dramatic advances in mass spectrometry technology, coupled with the availability of complete genome sequences to facilitate the assignment of peptides to proteins encoded by a genome, have led to efforts aimed at more rapidly identifying proteins capable of binding DNA motifs of interest. In fact, because of these advances, the need for development of an initial protein-DNA interaction assay and the need for protein purification before using mass spectrometry to identify proteins capable of binding a DNA motif may soon be eliminated. One such strategy uses a synthetic biotinylated double-stranded oligonucleotide containing the DNA motif of interest (with 5-10 bp of flanking sequence on each side). The biotinylated oligonucleotide is incubated with an appropriate nuclear extract, followed by a simple pulldown step using streptavidin-coated resin or magnetic beads. After washing, the proteins present in the pellet can be identified using MudPIT (Washburn et al. 2001; Fournier et al. 2007). The researcher can then sort through the collection of proteins to determine which ones might be responsible for the function of the DNA element. As a control, parallel experiments can be performed with a biotinylated oligonucleotide containing a mutation in the base pairs known to be critical for the function of the element. Proteins that bind the mutant 
oligonucleotide could be excluded from the list of candidates. Reed et al. (2008) provide one example of the successful use of this technique.

This strategy provides the researcher with several candidate proteins that might interact functionally with the DNA motif of interest. The availability of multiple candidates has both advantages and disadvantages. One important advantage is that the results are not limited to abundant proteins that yield a detectable EMSA complex (which may or may not be functionally relevant); MudPIT results reveal both abundant and less abundant proteins capable of binding the DNA element. On the other hand, it may take considerable time and effort to sort through the candidates. Some proteins will likely be identified that have a very low probability of being important transcription factors, based on their known functions or known properties of binding nucleic acids with little specificity. Attention can therefore be focused on proteins with domains commonly found in sequence-specific proteinDNA interactions (e.g., zinc fingers, homeodomains, Ets domains). ChIP experiments, expression studies, and an evaluation of the specific base pairs recognized by each candidate protein can then be used to determine which candidates are most viable. In some instances, a researcher using this MudPIT strategy may identify the functionally important protein quite rapidly. However, in other instances, the time required to sort through the many candidates may be comparable to, or may exceed, the time required to purify an attractive DNA-binding protein that originally was identified and characterized by EMSA.

\section{DEVELOPMENT OF A PROTEIN-DNA INTERACTION ASSAY FOR CRUDE CELL LYSATES}

Although the new mass spectrometry strategies being developed do not require initial use of an in vitro protein-DNA interaction assay, such an assay will eventually be needed to characterize the candidate DNA-binding proteins that are identified. In addition, because of the challenge of sorting the proteins identified in a MudPIT analysis, it may be preferable to pursue DNA-binding proteins using conventional protein-DNA interaction assays and then purifying attractive DNA-binding activities that are identified. Finally, even if an initial TRANSFAC or JASPAR database search suggests that a particular protein is capable of binding the DNA motif, a protein-DNA interaction assay is still needed to confirm and further characterize the predicted interaction. We therefore describe in detail the development of these assays.

\section{Standard Methods for Detecting Protein-DNA Interactions}

Several methods have been used to detect and characterize protein-DNA interactions in vitro, including EMSA, DNase I footprinting, exonuclease III footprinting, southwestern blotting, various chemical protection and interference assays, and ultraviolet cross-linking. However, EMSA is by far the most frequently used, largely because it is technically the easiest and is often the most sensitive. The second most common assay is DNase I footprinting, although its use is rapidly declining; its main advantage of allowing a researcher to localize a protein-binding site within a large DNA fragment is becoming less significant. We largely limit our discussion in this article to these two techniques.

\section{Basic EMSA and DNase I Footprinting Strategies}

Briefly, the EMSA is based on the principle that a protein-DNA complex migrates through a native gel more slowly than free DNA, with the mobility of the complex determined by the size, shape, charge, and multimeric state of the protein. Thus, proteins within a crude cell extract that specifically recognize a given control element can be identified by incubating a small radiolabeled DNA fragment with the extract to allow the formation of protein-DNA complexes. The mixture is then electrophoresed through a native polyacrylamide gel, which separates the free radiolabeled probe DNA from the molecules bound by proteins. The free and bound DNA molecules are detected by autoradiography or Phosphorimager analysis. 
For the DNase I footprinting assay, a radiolabeled DNA probe is first incubated with a cell extract to allow the formation of protein-DNA complexes. An important difference between this binding reaction and that used for the EMSA is that the double-stranded, radiolabeled DNA probe used for footprinting must be labeled on only one strand and on only one end. (In contrast, for the EMSA, the locations and number of radiolabeled nucleotides are unimportant.) The solution containing probe and extract is then treated with a limiting concentration of DNase I for a short, defined period of time. The DNase I treatment parameters are chosen so that each probe molecule is cleaved, on average, only once. Because cleavage is largely random, it will generate a nested set of DNA molecules varying in length from mono- and dinucleotides to full-length molecules that have not been cleaved. The DNA fragments are analyzed by denaturing PAGE followed by autoradiography or Phosphorimager analysis. If appropriate DNase I digestion parameters are used, the sample lacking the cell extract will yield a ladder of bands extending from the bottom to the top of the image. However, if a protein in the extract can bind a specific DNA sequence found on the probe, it will protect nucleotides within that region from digestion. On the resulting image, the protection will appear as a region devoid of bands (or a region containing very weak bands) relative to those observed in the absence of extract. The protected region is referred to as a DNase I footprint. DNA sequencing markers electrophoresed adjacent to the footprinting reactions allow a determination of the nucleotides protected by the protein.

\section{Differential Detection of Proteins by EMSA and DNase I Footprinting}

Although many proteins can be detected by both EMSA and DNase I footprinting, some DNAbinding proteins can be detected by only one of these assays. The precise reason has not been determined for most of the examples in which a protein is detected by only one of the two assays. However, a summary of the key differences between the two assays can provide insight into the possible explanations (Fig. 1; Table 1).

First and foremost, the EMSA is more sensitive and can reveal a specific protein-DNA complex even when the protein is at a low concentration within the extract. This is because a protein bound to only a few percent of the probe molecules still results in a complex that migrates more slowly

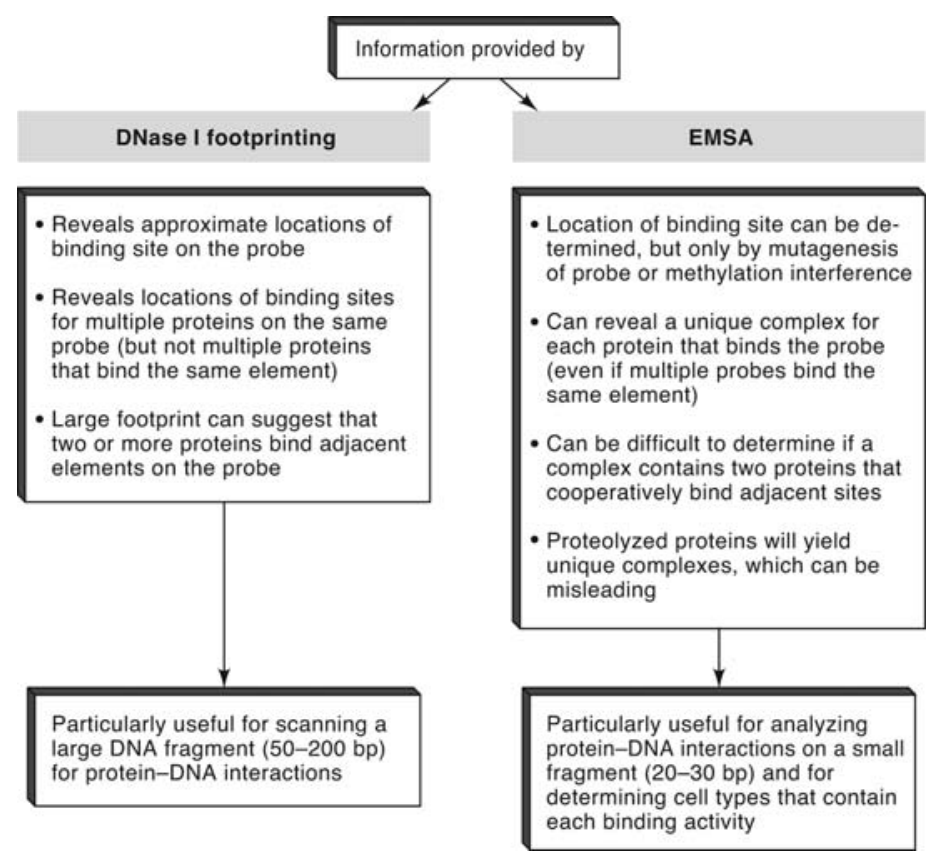

FIGURE 1. Information provided by DNase I footprinting and EMSA. 
TABLE 1. Characteristic differences between the EMSA and DNase I footprinting assays

\begin{tabular}{ll}
\hline EMSA & \multicolumn{1}{c}{ DNase I footprinting } \\
\hline $\begin{array}{l}\text { Assay is effective when protein is at low concentration. } \\
\text { Protein-DNA interaction must survive gel } \\
\text { electrophoresis. }\end{array}$ & $\begin{array}{l}\text { Assay is effective primarily when protein is at high concentration. } \\
\text { Binding must be stable only in solution. }\end{array}$ \\
$\begin{array}{l}\text { Large amount of nonspecific competitor can disrupt } \\
\text { specific interactions. }\end{array}$ & $\begin{array}{r}\mathrm{MgCl}_{2} \text { and } \mathrm{CaCl}_{2}, \text { which are required for DNase I activity, can } \\
\text { disrupt specific interactions. }\end{array}$ \\
\hline
\end{tabular}

than the free probe. If the specific activity of the probe is sufficiently high, this complex can easily be detected by autoradiography or Phosphorimager analysis. In contrast, to detect a protein-DNA complex by DNase I footprinting, a majority of the probe molecules must be bound by the protein. Efficient binding is needed because the basic footprinting procedure does not separate the bound and free probe molecules. On the gel image, bands must be diminished considerably to yield a convincing footprint. If only $50 \%$ of the probe molecules are bound by protein, for example, the intensities of the protected bands will be diminished by only twofold relative to the bands observed in the absence of extract. This small difference is usually difficult to evaluate. Because of this difference in sensitivity, some proteins-primarily those present at low concentrations within an extract—may be detectable by EMSA but not by DNase I footprinting.

A second key difference between the two assays is that DNase I footprinting is performed entirely in solution, whereas the EMSA requires the protein-DNA interaction to be maintained during gel electrophoresis. Few proteins bind DNA with dissociation rates that are compatible with the typical 2-4-h electrophoresis time period. For most proteins, the success of the EMSA relies on a process referred to as "caging," in which the gel matrix keeps the protein and DNA in close proximity to each other when they dissociate during electrophoresis, allowing them to reassociate rapidly and migrate through the gel as a complex. Although the caging process facilitates the analysis of many protein-DNA complexes, some complexes appear to be poorly maintained. Evidence that a complex does not tolerate gel electrophoresis is usually provided by the observation that a protein yields strong protection in a DNase I footprinting assay, whereas the same amount of protein and probe show a weak or undetectable EMSA complex.

A third difference between the EMSA and DNase I footprinting assays is noteworthy: the amount of nonspecific competitor DNA typically needed for the assay when analyzing a crude cell extract. In both assays, an excess of a competitor DNA such as poly $(\mathrm{dI}: \mathrm{dC})$ or poly(dA:dT) is usually included to prevent nonspecific and low-specificity DNA-binding proteins from binding the probe and obscuring the specific complexes. For DNase I footprinting reactions, $1 \mu \mathrm{g}$ of poly $(\mathrm{dI}: \mathrm{dC})$ competitor can usually be used with 10-100 $\mu \mathrm{g}$ of crude cell extract. In contrast, $2-8 \mu \mathrm{g}$ of poly(dI:dC) is often needed for EMSA reactions with 5-10 $\mu$ g of crude extract. Relatively high concentrations of competitor are needed for EMSA reactions because modest amounts of nonspecific nucleic-acid-binding proteins can lead to a smear of radioactive probe on the gel image, which prevents detection of the desired complexes. With the DNase I footprinting assay, the nonspecific binding proteins are distributed throughout the probe and are therefore less likely to affect significantly the detection of specific protein-DNA complexes. This difference can influence the degree of success of each assay if the DNA-binding protein of interest possesses a moderate affinity for the competitor DNA; the high concentrations of competitor used for EMSA reactions may compete with the probe for binding of the protein of interest, diminishing or abolishing the specific protein-DNA complex. A fourth difference is that the DNase I footprinting assay requires the addition of $\mathrm{MgCl}_{2}$ and $\mathrm{CaCl}_{2}$ for DNase I activity. Although most protein-DNA interactions are unaffected or even enhanced by the resulting ions, some interactions may be weakened.

In light of these differences, it should not be surprising that the activities of some DNA-binding proteins are more easily detected with one of the two assays. If a protein is detected only by EMSA, it usually is not sufficiently concentrated to bind the high percentage of probe molecules required for a DNase I footprint. For example, nuclear factor $-\kappa \mathrm{B}(\mathrm{NF}-\kappa \mathrm{B})$ is much easier to detect by EMSA than by 
M.F. Carey et al.

DNase I footprinting because of its relatively low concentration in nuclear extracts. In contrast, Sp1 and Ikaros originally were easier to detect by DNase I footprinting, until conditions were developed that allowed their successful detection by EMSA (Dynan and Tjian 1983; Lo et al. 1991; Hahm et al. 1994).

For most researchers, it is preferable to test a variety of EMSA conditions than to use DNase I footprinting as a routine assay. One of the early advantages of DNase I footprinting was that, when searching for proteins capable of binding a large promoter or enhancer fragment, it provided information about the location of the interaction on the probe. Now that researchers generally work with short synthetic oligonucleotides, this is less of an issue. DNase I footprinting is still useful if a researcher finds a need to search for proteins capable of binding a relatively long DNA fragment or if a researcher is interested in determining whether a pure protein can bind cooperatively to multiple sites on a DNA fragment that is too large for use in EMSA experiments.

\section{Electrophoretic Mobility-Shift Assay}

As described above, the EMSA is a common and relatively straightforward method for studying protein-DNA interactions. In this section, we focus on specific issues that must be considered when using EMSA to initially identify and characterize proteins within crude extracts that bind a defined DNA element.

\section{Radiolabeled Probe Design}

The design of the radiolabeled EMSA probe can influence the quality of the results obtained. For many proteins detected in crude extracts, double-stranded oligonucleotides of $\sim 20-25$ bp yield consistent, high-quality results. For example, EMSA experiments to detect NF- $\kappa B$ complexes are often performed with 19-bp oligonucleotides (Pierce et al. 1988). An oligonucleotide probe should minimally contain $5-10 \mathrm{bp}$ on each side of the functionally defined control element because the adjacent sequences, although not specifically recognized by the protein, may be required for a stable protein-DNA interaction.

To prepare the double-stranded fragment, two single-stranded oligonucleotides can be synthesized and annealed (see Ausubel et al. 1994, Unit 6.4). Alternatively, one long oligonucleotide containing both complements of the recognition sequence can be prepared, allowing self-annealing with an intervening loop. Crude desalted synthetic oligonucleotides can be radiolabeled either by $5^{\prime}$ end-labeling with $\left[\gamma_{-}{ }^{32} \mathrm{P}\right] \mathrm{ATP}$ and T4 polynucleotide kinase (see Sambrook and Russell 2001, p. 7.78) or by polymerization from a $3^{\prime}$ recessed end using $\alpha{ }^{32}$ P-labeled nucleoside triphosphates and Escherichia coli DNA polymerase Klenow fragment (Ausubel et al. 1994, Unit 3.5; Sambrook and Russell 2001, p. 10.30). The latter method has the advantage of labeling only those oligonucleotides that have annealed into a double-stranded molecule, as Klenow requires a template for nucleotide incorporation.

For some DNA-binding proteins, short oligonucleotides of 20-25 bp do not support stable protein-DNA interactions, making it necessary to use longer probes. The failure of short probes could result from the protein's requirement for a longer stretch of adjacent DNA for stable binding. Alternatively, short probes containing a particular recognition sequence might anneal improperly, dissociate during the binding reaction, or possess secondary structures that prevent binding. Several methods can be used to generate longer probes, one of which is simply to use longer synthetic oligonucleotides. Another method is to excise and radiolabel a restriction fragment from a plasmid containing the DNA element.

Polymerase chain reaction (PCR) with radiolabeled primers can also be used to prepare EMSA probes (Ausubel et al. 1994, Unit 15.2). One advantage of PCR is that wild-type and mutant probes can be prepared with similar specific activities if the same radiolabeled primer is used on both templates. If a series of mutations in a control element has been generated for a mutant analysis in a transfection assay, the same two PCR primers can be used to prepare probes containing the wildtype sequence and each mutant sequence for EMSA (e.g., see Emami et al. 1997). 


\section{Extract Preparation}

Most studies of in vitro protein-DNA interactions use nuclear extracts prepared according to the method of Dignam et al. (1983). Variations of the Dignam method that are better suited for small quantities of cells have also been developed. Briefly, cultured cells are swelled in a hypotonic buffer and then lysed by physical disruption with a Dounce homogenizer. An alternative method involves lysis with a low concentration of the nonionic detergent Nonidet P-40. With either physical or detergent lysis, the nuclei from most mammalian cell types remain reasonably intact and can be separated from the cytoplasmic proteins by low-speed centrifugation. To extract most DNA-binding proteins from the nuclei, the nuclear pellet is resuspended and stirred in a buffer containing a high concentration of $\mathrm{NaCl}$ or $\mathrm{KCl}$. Dignam and Roeder determined empirically that a $\mathrm{NaCl}$ concentration of $0.42 \mathrm{M}$ was optimal for the preparation of nuclear extracts that support in vitro transcription from the adenovirus major late promoter. This same salt concentration is used by most investigators to prepare nuclear extracts for the analysis of DNA-binding proteins. However, it may be beneficial to vary the salt concentration used for extract preparation when pursuing new DNA-binding activities. Lower salt concentrations can prevent inhibitors and nonspecific binding proteins from being extracted, increasing the success of the assay. Higher salt concentrations might be required for efficient extraction of a protein of interest.

If attempts to detect DNA-binding activities by EMSA fail, the nuclear extract can be further concentrated or fractionated to enhance the probability of success. The protein concentration of the extract can be increased by ammonium sulfate precipitation followed by extensive dialysis. A DNA-binding activity of interest may be detected more readily using a more concentrated extract. Dignam and Roeder found that extract precipitated using 53\% ammonium sulfate was optimal to enhance the activity of in vitro transcription extracts, but a different percentage may be optimal for a particular DNA-binding protein.

A second strategy to improve the detection of DNA-binding activities is to fractionate the extract (Ausubel et al. 1994, Unit 10.10). The negatively charged affinity resins heparin agarose and heparin sepharose are useful for this purpose. If extracts are applied to columns containing these resins in a buffer containing $0.1 \mathrm{M} \mathrm{KCl}$ or $\mathrm{NaCl}$, most DNA-binding proteins will elute with a $0.4 \mathrm{M}$ salt step. A $1 \mathrm{~m}$ salt step elution should also be performed in case the protein binds the resin with an unusually high affinity. The fractionation procedure separates the DNA-binding proteins from other proteins that might inhibit a binding reaction. Furthermore, the specific proteins within the 0.4 and $1 \mathrm{~m}$ salt fractions may be more concentrated than in the crude extracts. Therefore, DNA-binding activities may be detected in these fractions that were not detected in the crude extract.

If all attempts to detect candidate binding activities fail using nuclear extracts, whole-cell extracts, cytoplasmic extracts, or other nuclear extract preparation methods can be tested (Gorski et al. 1986; Shapiro et al. 1988; Soeller et al. 1988). Some nuclear proteins leak into the cytoplasmic fraction during nuclear extract preparation, leading to the possibility that the DNA-binding activity may be more abundant in a cytoplasmic or whole-cell extract.

\section{Competitor DNA and Other Components of Binding Reactions}

The nonspecific competitor DNA added to the binding reaction can be critical for the success of an EMSA experiment, especially when a crude nuclear extract is used. The competitor DNA prevents nonspecific and low-specificity nucleic acid-binding proteins from interacting stably with the radiolabeled probe. In the absence of an appropriate nonspecific competitor, numerous proteins within the extract (including abundant RNA-binding proteins) will bind the probe, leading to a radioactive smear on the gel image. Increased concentrations of competitor sequester more protein molecules from the radiolabeled probe, leaving primarily those proteins that have the highest specific affinity for the probe relative to the unlabeled competitor. Although high competitor concentrations often enhance the quality and clarity of the results, they also increase the probability that the relevant DNAbinding protein will be sequestered from the labeled probe. 
The most frequently used nonspecific competitor is poly(dI:dC). However, other competitors, including poly (dG:dC), poly(dA:dT), or sheared genomic DNA from E. coli or salmon sperm, may yield better (or at least different) results. If the protein of interest binds the competitor with high affinity, it might not yield a detectable complex on the EMSA gel. Instead, complexes containing proteins that are not relevant to the function of the control element may be observed if those proteins bind the competitor with a lower affinity. Therefore, it can be beneficial to test different competitors when attempting to identify candidates for the relevant regulator of a DNA element.

In addition to the competitor DNA, other components of the binding reactions can be varied to enhance detection of protein-DNA complexes, such as the concentration of monovalent or divalent cations. Some protein-DNA complexes may benefit from inclusion of a low concentration (e.g., $0.01 \%$ ) of the nonionic detergent Nonidet P-40 in the binding reaction. Others may benefit from inclusion of polyvinyl alcohol (2\%), which can increase the effective concentration of proteins in the extract. For additional recommendations for optimizing binding reactions for EMSA experiments, see Ausubel et al. (1994, Unit 12.2).

\section{Gel Electrophoresis Conditions}

When developing an EMSA for a DNA element of interest, pilot experiments with different gel electrophoresis buffers are strongly recommended, as detection of some protein-DNA complexes depends on the specific buffer used. The three most common EMSA gel buffers are Tris-borateEDTA, Tris-acetate-EDTA, and Tris-glycine. Inclusion of glycerol (5\%) can also enhance the detection of some binding activities. Furthermore, electrophoresis can be performed at room temperature or at $4^{\circ} \mathrm{C}$. For more information about different gel electrophoresis buffers, see Ausubel et al. (1994, Unit 12.2).

\section{Design of Initial Experiments and Interpretation of Results}

Initial EMSA experiments performed with a radiolabeled probe containing a control element of interest can result in the detection of one or more protein-DNA complexes on the gel image. Initial experiments are usually performed with a few different extract amounts (between 2 and $20 \mu \mathrm{g}$ ) and, for each extract amount, different amounts of nonspecific competitor $(1-10 \mu \mathrm{g})$. If complexes are not detected, the binding reaction and gel conditions can be modified as noted above. For each complex detected, two questions are initially addressed: (1) Is the protein-DNA complex specific for the probe being tested? (2) Is there a significant probability that the complex is responsible for the in vivo function of the DNA element in the context of the endogenous control region?

These questions are generally addressed by additional EMSA experiments performed in the presence of specific competitor DNAs or with mutant probes. The most straightforward information is usually provided by comparing the complexes obtained using a radiolabeled wild-type probe with those obtained using a radiolabeled mutant probe. If a protein-DNA complex is not observed with a probe containing nucleotide substitutions in the control element of interest, the protein-DNA interaction almost certainly involves those nucleotides. This answers both questions posed above, at least in part, by demonstrating that the interaction is sequence-specific and involves functionally relevant nucleotides. Further evidence that the complex might be responsible for the function of the control element can be obtained by preparing probes that contain other substitution mutations. If nucleotide substitutions that selectively disrupt the function of the control element (e.g., in a transfection experiment) correspond to those that disrupt the EMSA complex, the probability that the complex is functionally relevant is enhanced.

As with all standard EMSA experiments, the mutant analysis should be performed with low concentrations of probe (1-10 fmoles per reaction) and protein concentrations that yield complexes within a linear range (i.e., $1-50 \%$ of the probe associated with protein). Comparisons using wild-type and mutant probes should be performed with radiolabeled probes possessing similar specific activities. If the mutant probe has a lower specific activity than the wild-type probe, the protein-DNA complex will appear to be less abundant, even if the mutation has no effect on the affinity of the 
interaction. Unfortunately, the relative affinities of a protein for wild-type and mutant sequences cannot be determined simply by comparing the abundance of a complex formed with wild-type and mutant probes; slight decreases in affinity can lead to dramatic decreases in the amount of complex detected if the complex is not sufficiently stable to survive gel electrophoresis. Thus, if a mutation diminishes the abundance of a complex, one can conclude only that the protein has a lower affinity for the mutant probe than for the wild-type probe, but the magnitude of the difference cannot be discerned. On the other hand, if a mutation has no effect on complex formation, the protein most likely binds the wild-type and mutant probes with comparable affinities. Overall, despite the lack of quantitative data, a comparison of wild-type and mutant probes can provide valuable information about (1) the specificity of an interaction, (2) the nucleotides required for the interaction, and (3) the relationship between the required nucleotides and those required for function of the DNA element.

Another approach to examine the specificity and functional relevance of a protein-DNA complex is to perform competition experiments with unlabeled wild-type and mutant DNA fragments, such as annealed oligonucleotides, PCR products, or restriction fragments. For the results of competition experiments to be meaningful, the wild-type and mutant competitors must be quantified carefully to ensure that their concentrations are comparable. Competition experiments are performed by including a radiolabeled wild-type probe in a series of binding reactions containing increasing concentrations of unlabeled wild-type or mutant competitor DNAs. Radiolabeled complex abundance decreases in the presence of unlabeled competitor because the DNA-binding protein distributes itself randomly among the probe and competitor molecules. Because the wild-type competitor has a higher affinity for the protein than does the mutant competitor, lower concentrations are required to reduce the abundance of the complex.

Valuable information about the sequence specificity and functional relevance of a protein-DNA interaction can be obtained by comparing a wild-type competitor to a series of mutant competitors. If an EMSA complex is diminished more by the wild-type competitor than by a mutant, the protein must be binding DNA in a sequence-specific manner. If the mutations that diminish competition correspond to the mutations that reduce the function of the DNA element (e.g., in a transfection assay), the results suggest that the protein-DNA interaction may be functionally relevant.

The principal advantage of assessing specificity by using mutant competitor DNAs instead of radiolabeled mutant probes is that the former provides more accurate information about the relative affinities of a protein for the wild-type and mutant sequences. As explained above, a comparison of radiolabeled wild-type and mutant probes provides limited information about relative binding affinities. Competition experiments are not subject to the same caveat because the key events involved in the comparison of wild-type and mutant sequences occur in solution during the initial binding reaction; gel electrophoresis is performed after the competition has been completed. If the affinity of the protein for a mutant sequence is twofold less than its affinity for a wild-type sequence, then a twofold higher concentration of the mutant competitor relative to the wild-type competitor should reduce complex abundance by a given amount. Thus, the competition strategy has greater potential to provide meaningful quantitative information about relative affinities.

Although the above considerations appear to be straightforward, the results of competition experiments can be complicated. For example, a twofold reduction often requires a large excess of wild-type competitor. This is because most EMSA experiments contain excess protein, despite the presence of free probe at the bottom of the gel. Thus, competition occurs only when the concentration of competitor is high enough to exceed the $K_{\mathrm{d}}$ of the interaction. Although this issue makes it difficult to predict the effect of a given concentration of wild-type competitor, it does not completely invalidate the comparison of wild-type and mutant competitors. Even if a 50-fold excess of the wild-type competitor is needed to achieve a twofold reduction in the abundance of a protein-DNA complex, the effect of the mutant competitor can provide insight into the relative affinities of the protein for the wild-type and mutant sequences. If a 50-fold excess of the mutant competitor results in a similar twofold reduction in the protein-DNA complex, one can conclude that the mutation has no significant effect on the affinity of the protein for the DNA. If a 100-fold excess of the mutant competitor is needed for a twofold reduction in the protein-DNA complex, the affinity of the protein for the mutant 
sequence is approximately twofold less than that of the wild-type sequence. Because the radiolabeled probe and competition strategies for assessing specificity and functional relevance of a protein-DNA interaction possess unique advantages and limitations, the use of both is strongly recommended.

\section{Analysis of EMSA Complexes by Methylation Interference}

The experiments discussed above provide information about the specificity and nucleotide requirements of protein-DNA interactions detected by EMSA. Another technique-methylation interference-can be coupled to the EMSA to provide information about the specific nucleotide contacts involved in the interaction. Briefly, this assay begins with an EMSA probe labeled on only one end, which, after labeling and purification, is modified on guanine bases with dimethylsulfate. For this assay to succeed, the protein-binding site generally must be at least 25 or $30 \mathrm{bp}$ from the radiolabeled end, with 15-20 bp of sequence following the binding site. Thus, radiolabeled probes used for methylation interference are generally longer than those used for conventional EMSAs. The concentration of dimethylsulfate and time of incubation are chosen to result in an average of one methylguanine per probe molecule. The modified probe is incubated with extract in a standard EMSA-binding reaction. DNA-binding proteins bind randomly to the modified and unmodified probe molecules. However, a protein may be incapable of binding probe molecules in which a guanine that must be in close contact with the protein is methylated. The EMSA gel is used to separate the free probe molecules from the protein-DNA complexes. Following autoradiography, the film and gel are aligned, allowing excision of polyacrylamide gel slices containing the free probe and the protein-DNA complexes. The DNAs are eluted from the two gel slices and are then incubated with piperidine, which cleaves each probe molecule at the nucleotide(s) containing methylguanine. The resulting DNA fragments are analyzed by PAGE, followed by autoradiography or Phosphorimager analysis. If methylation of a particular guanine prevents DNA binding, DNA fragments cleaved at that guanine will be absent in the sample derived from the protein-DNA complex but will be abundant in the sample derived from the free DNA probe. Because methyl groups are quite small, the inhibition of protein binding by a particular methylguanine residue suggests that the protein is in close proximity with that guanine.

In many respects, methylation interference results can provide information that is similar to that provided by the EMSA competition and mutant studies: They can show that binding is sequencespecific and can reveal the location of the binding site. A limitation of the technique is that it provides only partial information about the nucleotides required for the protein-DNA interaction. A careful mutant analysis can provide much more detailed information, which can be correlated more effectively with the results of functional analyses. In addition, methylation interference can be technically challenging if the protein-DNA complex is not abundant. For weak complexes, it may not be possible to obtain compelling results, making the competition and mutant studies essential. For strong complexes, the use of all three approaches is recommended to provide as complete a picture as possible of the protein-DNA interaction. Additional techniques (not described here) can be used to model protein-DNA interactions more rigorously, but this detailed analysis is usually performed with recombinant proteins after the gene encoding the protein has been identified.

\section{Analysis of Previously Described Proteins in an EMSA Complex}

After the sequence specificity and nucleotide requirements for a protein-DNA interaction have been determined, it often is important to address the possibility that the protein responsible for the interaction has been described previously. The first step of this analysis is to search a binding-site database to determine whether the DNA element is similar to the recognition site for one or more known proteins. If candidate proteins are identified by the database search, the possibility that they are responsible for the EMSA complex can be evaluated using antibodies. Antibodies directed against many known DNA-binding proteins are available from a variety of commercial and academic sources. Otherwise, antibodies can be prepared against a synthetic peptide or a bacterially expressed fusion protein (see Harlow and Lane 1999). 
To determine whether an EMSA complex contains a known protein, an antibody can be added directly to the binding reaction or preincubated with the extract before addition of radiolabeled probe. Preincubation may permit more efficient formation of the antibody-antigen complex if the DNA-binding domain is recognized by the antibody. In such a case, the antibody may prevent DNA binding, leading to a reduction in the amount of protein-DNA complex observed on the EMSA gel image (Fig. 2A). If the antibody recognizes a domain that is distinct from the DNA-binding domain, it is more likely to "supershift" the complex, meaning that the complex will migrate more slowly through the EMSA gel because its molecular weight will be higher when bound to the antibody (Fig. 2B). An antibody can also stabilize a protein-DNA interaction by stabilizing the protein in a binding-competent conformation. This results in a supershifted band that is more abundant than the original band observed in the absence of antibody.

Antibodies are useful for determining the identity of a protein within an EMSA complex. However, they must be used with caution, primarily because most polyclonal and monoclonal antibody preparations are impure. The contaminants within the preparations can inhibit complex formation as effectively as a specific antibody-antigen interaction. Carefully designed controls must be included, particularly if the antibody inhibits, rather than supershifts, a complex. Appropriate controls for a polyclonal antibody are preimmune serum or a polyclonal preparation directed against an unrelated protein. It is also helpful to test the effect of an antibody preparation on an EMSA complex obtained with an unrelated probe bound by a protein that should not be recognized by the antibody. The specificity of the antibody effects can sometimes be greatly improved by purifying the antibodies by protein A-Sepharose or protein G-Sepharose chromatography or by antigen affinity chromatography (see Harlow and Lane 1999). If purified polyclonal antibodies are used in conjunction with the EMSA experiments, control antibodies should be subjected to the same purification protocol and analyzed along with the test antibodies. If monoclonal antibodies are used, a control monoclonal antibody prepared by the same method and directed against an unrelated protein should be included.

If the antibodies supershift rather than inhibit the complex, the controls described above are somewhat less important. However, supershift results must be interpreted with equal caution. In some instances, the abundance of a protein-DNA complex will not be diminished by the antibody, even though a supershifted complex of weak or moderate intensity appears on the gel image (Fig. 2C). This result does not establish that the protein recognized by the antibody is a component of the original complex. Because some antibodies stabilize protein-DNA interactions (see above), the supershifted complex might correspond to a stabilized form of a complex that was not detected in the absence of antibody, unrelated to the original protein-DNA complex. Evidence that a complex contains a particular protein is provided only when the abundance of the complex decreases as the supershifted complex appears. Another caveat of antibody supershifts is that antibodies can promote cooperative binding of a protein to two adjacent sites.

If the antibody does not disrupt or supershift the complex, the protein recognized by the antibody may not be present within the complex. Alternatively, the antibody could be too dilute or might bind

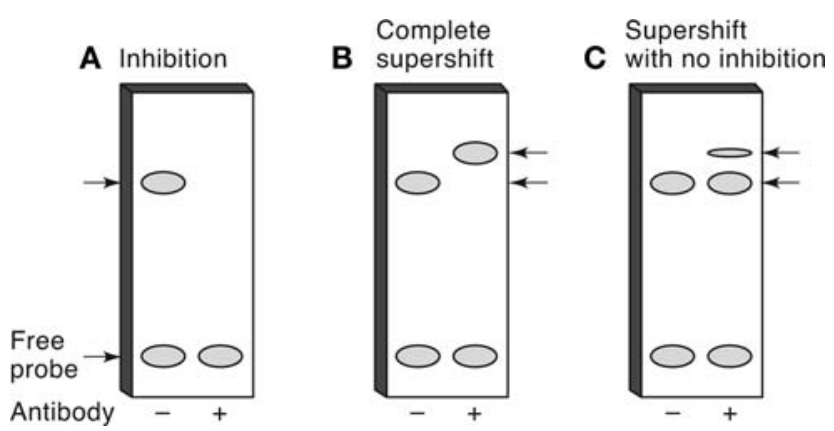

FIGURE 2. Effects of antibodies on EMSA complexes. 
M.F. Carey et al.

to the target protein with a low affinity. A positive control is needed, in which the antibody is shown to disrupt or supershift an EMSA complex known to contain the protein.

If the protein-DNA complex is convincingly disrupted or supershifted by an antibody against a known protein, the complex is likely to contain that protein. To test this hypothesis further, a cDNA for the protein can be obtained and analyzed. The full-length protein can often be produced by in vitro transcription/translation (Ausubel et al. 1994, Unit 10.17) and tested by EMSA side by side with the crude cell extract. If the complex detected with the crude extract contains only one protein, it should comigrate with the EMSA complex containing the in vitro-translated protein. If not, it may be necessary to reevaluate the antibody results. Alternatively, the protein may bind as a heterodimer with another protein or may bind cooperatively with a protein that recognizes an adjacent site on the probe. Knowledge of the general properties of the protein family, and detailed knowledge of the nucleotides within the probe required for complex formation, should allow these possibilities to be evaluated.

If these studies suggest that a known protein binds the control element of interest, the relevance of the protein for the function of the element can be evaluated. In contrast, if the results suggest that the protein has not been previously described, the identity of the protein and the gene encoding the protein can be pursued. A final possibility is that the complex contains a heterodimer of a known protein and a novel partner. To identify the unknown components, the proteins that form the complex may need to be purified.

\section{DNase I Footprinting}

The DNase I footprinting assay is described above briefly. In this section, specific issues are discussed that must be considered when using a DNase I footprinting assay with crude extracts to identify protein-DNA interactions.

\section{Radiolabeled Probe Design}

Two important issues are the length of the radiolabeled probe and the location of the relevant DNA element(s) within the probe. The probe must be radiolabeled on only one end and on only one strand. Such probes can be prepared by direct labeling of restriction fragments or by PCR with one radiolabeled primer and one unlabeled primer (Ausubel et al. 1994, Unit 15.2.6). Synthetic oligonucleotide probes are used less frequently for DNase I footprinting than for EMSAs because longer probes are generally preferred.

For DNase I footprinting with crude extracts, probes generally range from $\sim 100$ to several hundred base pairs in length (shorter probes are often used with recombinant proteins). It is important that the DNA element(s) of interest not be within $40 \mathrm{bp}$ of either end of the probe. One reason for this restriction is that some extracts contain abundant DNA end-binding proteins, which can obscure detection of a specific protein-DNA interaction at either end of a probe. Furthermore, accurate evaluations of DNase I footprinting results depend on detecting cleavage products on both sides of the protected region that are unaffected by protein binding; if the protected region is too close to the end of the probe, it may be difficult to detect unaffected cleavage products at one end.

To evaluate DNase I footprinting data accurately, it is also useful if the DNA elements of interest are within $\sim 200$ bp of the labeled end. Probes of 500 bp or more can be used successfully in the footprinting assay, but protein-DNA interactions that occur $>200 \mathrm{bp}$ from the radiolabel are often difficult to detect because of compression of bands at the top of the gel. Considering these restrictions, the DNase I footprinting procedure can usually provide information about protein-DNA interactions that occur within a region of $\sim 160 \mathrm{bp}$, corresponding to a region that is $40-200 \mathrm{bp}$ from the labeled end of the probe. Thus, if the control region being analyzed is $\sim 300 \mathrm{bp}$ in length, it may be possible to scan the entire region for specific protein-DNA interactions with only two probes.

To increase further the probability that the protein-DNA interaction of interest will be detected, an independent set of probes labeled on the opposite strand can be tested. Some protein-DNA interactions are detected more readily with a probe labeled on one strand than the other. Furthermore, 
analysis of both strands reduces the probability that an important protein-DNA interaction will occur in a region that is resistant to DNase I cleavage. DNase I nicks one strand of a double-stranded DNA fragment. This nicking occurs at many sites on a naked DNA probe, but it is not completely random. Thus, regions of $20 \mathrm{bp}$ or more that are resistant to DNase I nicking can be observed. If a relevant protein-DNA interaction occurs within such a region, it will not be detected. By testing two probes labeled on opposite strands, the probability that an interaction will be missed because a long stretch of DNA on one strand or the other is resistant to DNase I cleavage will be reduced.

It is important to note that the location of a protein-DNA interaction detected by DNase I footprinting is determined by comparing the protected region on the gel image to DNA size markers that are run in an adjacent lane. Radiolabeled restriction fragments can provide some indication of the distance from the labeled end of the probe to the protected region, but DNA sequencing markers provide more accurate information.

\section{Extract Preparation}

Preparation methods and strategies to optimize and concentrate extracts for DNase I footprinting are similar to those described for EMSA. However, for DNase I footprinting, it is more often necessary to concentrate extracts by ammonium sulfate precipitation or heparin-Sepharose chromatography. This is because a much larger percentage of the probe molecules must be bound by a specific protein to detect a footprint (see above).

\section{Binding Reactions}

In general, the binding reactions for DNase I footprinting with crude extracts are similar to those used for EMSA, but the concentrations of some of the components differ. The concentration of nonspecific competitor DNA such as poly $(\mathrm{dI}: \mathrm{dC})$ is generally lower, and the concentration of extract is generally higher in a DNase I footprinting assay. A standard concentration of poly (dI:dC) for DNase I footprinting with a crude extract is $1 \mu \mathrm{g}$ per $50-\mu \mathrm{L}$ binding reaction. Optimal crude extract concentrations range from 50 to $250 \mu \mathrm{g}$ in a $50-\mathrm{mL}$ reaction. It is particularly important to test different concentrations of extract. A concentration that is too high can result in probe degradation or dephosphorylation by proteins within the extract or complete resistance of the probe to DNase I cleavage.

DNase I cleavage reactions can be described from the perspective of analyzing purified recombinant DNA-binding proteins. The only notable difference when analyzing activities in crude extracts is that a higher concentration of DNase I may be required. Therefore, the optimal concentration of DNase I must be determined separately for control reactions lacking protein and for the reactions containing each concentration of extract. When the extract concentration in the reaction is increased, it may be necessary to increase the concentration of DNase I further. For each set of reaction conditions, the goal is to identify a DNase I concentration that yields bands of similar intensities toward the top and bottom of the gel image.

There are a few useful methods to improve DNase I footprinting results when using crude extracts. First, if protection is not observed at the DNA element of interest, the sensitivity of the assay often can be enhanced by adding $2 \%$ polyvinyl alcohol (from a $10 \%$ stock solution) to the binding reactions. This polymer increases the effective concentration of the proteins within the extract, which may enhance the efficiency of a relevant protein-DNA interaction. Second, more consistent results can sometimes be obtained if the binding reactions and DNase I digestions are performed on ice rather than at room temperature (unless the protein only binds DNA at room temperature or higher). For a given extract, both temperatures should be tested to determine whether one yields significantly better results than the other. A higher concentration of DNase I is required when the digestions are performed on ice because the rate of cleavage is reduced. For an investigator performing a DNase I footprinting experiment for the first time, it may beneficial to use a commercially available kit (e.g., Promega’s Core Footprinting System) that includes standard reagents and positive controls. 


\section{Interpretation of Initial Results}

DNase I footprinting results obtained with crude extracts often provide immediate and compelling evidence that sequence-specific DNA-binding proteins within the extract can bind specific regions of the probe. A reaction performed in the absence of DNA-binding proteins is run on the gel adjacent to the reactions performed in the presence of extract. To simplify interpretation of the data, the bands in the control lane and experimental lanes should be of similar intensity throughout the gel, except at specific regions of protection. If the general band intensities in the two lanes are significantly different, it may be necessary to alter the concentration of DNase I used in one of the reactions.

An additional useful control when analyzing crude extracts involves incubating the extract and probe in a typical binding reaction and then performing a mock nuclease digestion by adding $\mathrm{MgCl}_{2}$ and $\mathrm{CaCl}_{2}$, but no DNase I. This reaction provides information about cleavage products resulting from nucleases within the extract, which can cleave at sites different from those of DNase I, thereby complicating the interpretation of the results. Nucleases can be even more problematic if the standard binding buffer also contains $\mathrm{MgCl}_{2}$. Because most DNA-binding proteins do not require $\mathrm{Mg}^{++}$for binding, initial experiments should be attempted using buffers both with and without $\mathrm{MgCl}_{2}$. If substantial nuclease activity is detected in the extract, it may be necessary to fractionate the extract by column chromatography (e.g., heparin-Sepharose) before footprinting analysis to separate the nucleases from the majority of the DNA-binding proteins.

In addition to the cleavage sites that are protected from DNase I digestion by bound proteins, other sites can be hypersensitive to DNase I cleavage. Hypersensitivity is frequently observed near the edges of protected regions and results from conformational changes in the DNA on protein binding. At least one protein family, the Ets family, induces a characteristic hypersensitive site at the center of the protected region (Ernst et al. 1993). Hypersensitive sites can be highly informative because they can appear when only a small fraction of the probe molecules are bound by protein, whereas compelling protection appears only when the vast majority of the probe molecules are occupied. Thus, the presence of a hypersensitive site in a region that does not show protection suggests that a protein within the extract binds nearby, but the protein may not be sufficiently concentrated for protection. There are two alternative explanations for the presence of isolated hypersensitive sites: (1) A nuclease within the extract can lead to the appearance of hypersensitive cleavage sites because those sites may not be cleaved by DNase I in the control reaction that lacks extract or (2) structural alterations that enhance DNase I cleavage may occur when proteins bind to distant sites. For example, two proteins bound at sites that are distant from each other may interact, inducing structural alterations and enhanced DNase I cleavage in the intervening sequence.

Some of the issues discussed for interpreting initial EMSAs are relevant to DNase I footprinting, but others are less relevant. In particular, the specificity of a protein-DNA interaction is much easier to establish with DNase I footprinting. If a discrete footprint is observed, the protein-DNA interaction is specific for a sequence within the protected region; competition experiments and mutant studies generally are not needed to establish the specificity of the interaction. Experiments must be performed, however, to determine whether the protein-DNA interaction involves nucleotides that are relevant for the function of the control element. As with the EMSAs, the nucleotide requirements for the interaction can be addressed using radiolabeled mutants or by competition using wild-type or mutant oligonucleotides. The former strategy is generally preferred because it is much less susceptible to misinterpretation. If radiolabeled wild-type and mutant probes are prepared by PCR using the same radiolabeled primer, the probes will have comparable specific activities. If a protein-DNA interaction is disrupted by a mutation, the results provide strong evidence that the nucleotides mutated are required. In addition, because DNase I footprinting reactions are performed entirely in solution (i.e., the protein-DNA interaction does not need to survive a gel electrophoresis step), relative affinities determined by comparing radiolabeled wild-type and mutant probes can be as accurate, if not more so, than relative affinities determined by competition experiments.

As with EMSAs, antibodies can be used to determine if the protein responsible for a DNase I footprint has been described previously. However, the antibodies are useful only if they disrupt the 
DNA-binding activity of the protein, usually by binding the DNA-binding domain. Antibodies that do not disrupt DNA binding usually have no noticeable effect on DNase I footprinting, in contrast to the supershift observed in an EMSA.

It is important to emphasize that different types of information are provided by DNase I footprinting and the methylation interference assay, which can be coupled to the EMSA. The protected region observed during DNase I footprinting corresponds to the nucleotides that are inaccessible to DNase I cleavage when the protein is bound. Because DNase I is a protein with a substantial mass, and because the DNA-binding protein usually covers nucleotides adjacent to those that are directly contacted, the protected region almost always includes at least a few nucleotides on both sides of the critical nucleotides. The critical nucleotides are usually toward the center of the protected region, but, in the absence of mutant or competition studies, they are difficult to predict. In contrast, the methylation interference assay provides more precise information about the protein-DNA contacts because the DNA is modified by a small methyl group before protein binding. Methyl groups interfere with protein binding only if they modify a guanine that directly contacts the protein or is in very close proximity to an inflexible region of the protein.

\section{REFERENCES}

Ausubel FM, Brent RE, Kingston E, Moore DD, Seidman JG, Smith JA, Struhl K. 1994. Current protocols in molecular biology. Wiley, New York.

Dignam JD, Lebovitz RM, Roeder RG. 1983. Accurate transcription initiation by RNA polymerase II in a soluble extract from isolated mammalian nuclei. Nucleic Acids Res 11: 1475-1489.

Dynan WS, Tjian R. 1983. The promoter-specific transcription factor Sp1 binds to upstream sequences in the SV40 early promoter. Cell 35: 79-87.

Emami KH, Jain A, Smale ST. 1997. Mechanism of synergy between TATA and initiator: Synergistic binding of TFIID following a putative TFIIAinduced isomerization. Genes Dev 11: 3007-3019.

Ernst P, Hahm K, Smale ST. 1993. Both LyF-1 and an Ets protein interact with a critical promoter element in the murine terminal transferase gene. Mol Cell Biol 13: 2982-2992.

Fournier ML, Gilmore JM, Martin-Brown SA, Washburn MP. 2007. Multidimensional separations-based shotgun proteomics. Chem Rev 107: 3654-3686.

Gorski K, Carneiro M, Schibler U. 1986. Tissue-specific in vitro transcription from the mouse albumin promoter. Cell 47: 767-776.

Hahm K, Ernst P, Lo K, Kim GS, Turck C, Smale ST. 1994. The lymphoid transcription factor LyF-1 is encoded by specific, alternatively spliced mRNAs derived from the Ikaros gene. Mol Cell Biol 14: 7111-7123.

Harlow E, Lane D. 1999. Using antibodies: A laboratory manual. Cold Spring Harbor Laboratory Press, Cold Spring Harbor, NY.

Lo K, Landau NR, Smale ST. 1991. LyF-1, a transcriptional regulator that interacts with a novel class of promoters for lymphocyte-specific genes. Mol Cell Biol 11: 5229-5243.

Pierce JW, Lenardo M, Baltimore D. 1988. Oligonucleotide that binds nuclear factor NF- $\mathrm{kB}$ acts as a lymphoid-specific and inducible enhancer element. Proc Natl Acad Sci 85: 1482-1486.

Reed DE, Huang XM, Wohlschlegel JA, Levine MS, Senger K. 2008. DEAF-1 regulates immunity gene expression in Drosophila. Proc Natl Acad Sci 105: 8351-8356.

Sambrook J, Russell DW. 2001. Molecular cloning: A laboratory manual, 3rd ed. Cold Spring Harbor Laboratory Press, Cold Spring Harbor, NY.

Shapiro DJ, Sharp PA, Wahli WW, Keller MJ. 1988. A high-efficiency HeLa cell nuclear transcription extract. DNA 7: 47-55.

Soeller WC, Poole SJ, Kornberg T. 1988. In vitro transcription of the Drosophila engrailed gene. Genes Dev 2: 68-81.

Washburn MP, Wolters D, Yates JR 3rd. 2001. Large-scale analysis of the yeast proteome by multidimensional protein identification technology. Nat Biotechnol 19: 242-247. 


\title{
Experimental Strategies for the Identification of DNA-Binding Proteins
}

\author{
Michael F. Carey, Craig L. Peterson and Stephen T. Smale
}

Cold Spring Harb Protoc; doi: 10.1101/pdb.top067470

\begin{tabular}{rc}
$\begin{array}{r}\text { Email Alerting } \\
\text { Service }\end{array}$ & Receive free email alerts when new articles cite this article - click here. \\
\hline Subject & Browse articles on similar topics from Cold Spring Harbor Protocols. \\
Categories & Bioinformatics/Genomics, general (192 articles) \\
& DNA:Protein Interactions (74 articles) \\
& Electrophoresis (57 articles) \\
& Electrophoresis, general (130 articles) \\
& Mass Spectrometry (78 articles) \\
& Molecular Biology, general (1293 articles) \\
& Sequence Database Searching (38 articles) \\
\hline
\end{tabular}

